\title{
Learning and Classifying Under Hard Budgets
}

\author{
Aloak Kapoor and Russell Greiner* \\ Department of Computing Science, \\ University of Alberta, Edmonton, AB T6J 2E8 \\ \{aloak, greiner\}@cs.ualberta.ca
}

\begin{abstract}
Since resources for data acquisition are seldom infinite, both learners and classifiers must act intelligently under hard budgets. In this paper, we consider problems in which feature values are unknown to both the learner and classifier, but can be acquired at a cost. Our goal is a learner that spends its fixed learning budget $b_{L}$ acquiring training data, to produce the most accurate "active classifier" that spends at most $b_{C}$ per instance. To produce this fixed-budget classifier, the fixedbudget learner must sequentially decide which feature values to collect to learn the relevant information about the distribution. We explore several approaches the learner can take, including the standard "round robin" policy (purchasing every feature of every instance until the $b_{L}$ budget is exhausted). We demonstrate empirically that round robin is problematic (especially for small $b_{L}$ ), and provide alternate learning strategies that achieve superior performance on a variety of datasets.
\end{abstract}

\section{Introduction}

While a doctor may have the option of using a wide variety of medical tests (including MRIs, blood work, etc.) to diagnose a patient, many medical plans involve capitation payments that restrict the per-patient cost of medical diagnosis and treatment. These physicians can only consider diagnostic strategies that spend at most a specified amount; they would clearly want to use the most accurate such strategy. In general, these strategies can operate sequentially: e.g. first performing test $\operatorname{Blood}_{7}\left(\right.$ at cost $\left.C\left(\operatorname{Blood}_{7}\right)\right)$, then using this information to decide on the next action; perhaps performing $\operatorname{Liver}_{3}$ if $\mathrm{Blood}_{7}$ was positive, but performing Urine 2 if Blood $_{7}$ was negative, and so forth. Once the total cost of the tests performed reaches the capitation amount $b_{C}$ (i.e. if $C\left(\right.$ Blood $\left._{7}\right)+C\left(\right.$ Urine $\left.\left._{2}\right)+\cdots=b_{C}\right)$, the strategy must stop collecting information and render a decision - e.g. "Cancer $=$ true". We call such a strategy a "bounded active classifier" 1 .

Earlier results [1] have shown that one can PAC-learn the decision-theoretic optimal "bounded active classifier" $\mathrm{BAC}^{*}=\arg \min _{b}\left\{\operatorname{error}(b) \mid b \in \operatorname{cost}-b_{C^{-}}\right.$ active classifiers $\}$, assuming the learner has no a priori resource bound - i.e.

\footnotetext{
* Both authors wish to thank NSERC and iCORE for their generous support. We also thank Dan Lizotte and Omid Madani for insights on various related problems, and the anonymous reviewers for their comments. Russell Greiner also thanks the Alberta Ingenuity Centre for Machine Learning.
} 
it can purchase every feature of as many instances as necessary. Of course, if we are charging the classifier (read "physician") for each feature, it seems strange to provide this information for free to the learner (think "experimental designer"). This paper extends those earlier results by investigating the challenge of learning this $\mathrm{BAC}^{*}$ when the learner has a fixed budget to spend acquiring the relevant training data - i.e., when the learner can spend only a total of $b_{L}$ to produce the best classifier that can spend only $b_{C}$ per instance. Thus, we investigate the problem of budgeted learning a bounded active classifier.

In Sect. 2, we introduce the formal framework for budgeted learning a bounded active classifier, highlight the simplifying assumptions we make, and derive complexity results that show our task is NP-hard in general. Section 3 demonstrates how to improve the running time of the (intractable) optimal algorithm, while Sect. 4 discusses a variety of tractable algorithms that attempt to find good approximate solutions to the problem. Section 5 describes the loss functions that are required by some of our approaches, and Sect. 6 gives empirical results that compare the proposed algorithms. Finally, Sect. 7 reviews related literature and Sect. 8 summarizes our contributions. The proofs, and other information about these studies, all appear in the website [5].

\section{Formal Description}

The "budgeted bounded-active-classifier learner", BBACL, is given the (nonnegative) cost $C\left(X_{i}\right) \in \mathbb{R}^{+}$of acquiring each individual feature $X_{i}$ of any single specified instance 1 and the loss matrix $L=\left[\ell_{i, j}\right]$ whose $(i, j)$ element specifies the penalty for returning the class $c_{i}$ when the true class is $c_{j}$; by convention we assume $\ell_{i, i}=0$ and $\ell_{i, j}>0$ for $i \neq j$. BBACL also knows the total amount the learner can spend $b_{L} \in \mathbb{R}^{+}$, and how much the resulting active classifier can spend per instance $b_{C} \in \mathbb{R}^{+}$.

At any time, the BBACL can see the current $m \times(r+1)$ "tableau", whose rows each correspond to an instance $i \in\{1, \ldots, m\}$ and whose first $r$ columns each correspond to a feature, and whose $r+1$ st column is the class label. Initially, only the class label is specified; the other $m \times r$ entries are all unknown. In general, we will let $x_{i}^{(j)}$ refer to the initially unknown value of the $i$ th feature of the $j$ th instance. At any point, BBACL can perform the $(i, j)$ "probe" to determine the value of $x_{i}^{(j)}$, at cost $C\left(X_{i}\right)$. This also reduces BBACL's remaining budget from $b_{L}$ to $b_{L}-C\left(X_{i}\right)$. Once this budget reaches zero, BBACL stops collecting information and returns a bounded active classifier BAC, which corresponds to a decision tree of bounded depth [2].

The score of any BAC $B$ is its expected misclassification error:

$$
Q(B)=\sum_{\mathbf{x}, y} P(\mathbf{x}, y) L(B(\mathbf{x}), y) .
$$

\footnotetext{
${ }^{1}$ We assume that these costs are independent of each other, both within and across instances. Moreover, if any test costs $C\left(X_{i}\right)=0$, we can simply gather that information for each instance and then consider the resulting reduced problem where $C\left(X_{i}\right)>0$ for all remaining $X_{i}$ s.
} 
Letting $A l l\left(b_{C}\right)$ be the set of all such active classifiers that spend at most $b_{C}$ per instance, our goal is the $\mathrm{BAC}$ from this set that minimizes this error:

$$
\mathrm{BAC}^{*}=\underset{B \in A l l\left(b_{C}\right)}{\arg \min } Q(B) .
$$

\subsection{Simplifying Assumptions}

For our work we will assume a constant misclassification $\operatorname{cost} \ell_{i j}=1$ for $i \neq j$ and $\ell_{i i}=0$. Our algorithms will need to estimate the probabilities over the values of the features of an instance $P\left(x_{i}^{(j)}\right)$ to decide which probe to perform. We will take a Bayesian stance by assuming there is a prior distribution over labeled instances, before seeing any data 2 As a simplification, we will make the Naïve Bayes assumption, which means the distribution of $x_{i}^{(j)}$ is independent of $x_{k}^{(j)}$ (for $k \neq i$ ) as we know the value of the class $y_{j} 3$ Hence, if instance $j$ is labeled with class + , we will model the distribution of its $i$ th feature $x_{i}^{(j)} \sim \operatorname{Dir}\left(\alpha_{1,+}^{(i)}, \ldots, \alpha_{w,+}^{(i)}\right)$ as a Dirichlet distribution with parameters $\alpha_{j,+}^{(i)}>0$, assuming $X_{i}$ has $\left|X_{i}\right|=w$ values [3]. These parameters are unrelated to the ones for negatively labeled instances $\alpha_{j,-}^{(i)}$ and also unrelated to the parameter values for other features $X_{h}$, for $h \neq i$. Initially, we will assume that each such distribution is uniform $\operatorname{Dir}(1, \ldots, 1)$. If we later see a sample $S$ with $29 Y=+$ instances with $X_{i}=+$ and $14 Y=+$ instances with $X_{i}=-$, the posterior distribution for $x_{i}^{(j)}$ for a new $Y=+$ instance would be $\operatorname{Dir}(1+29,1+14)$. The mean probability for $X_{i}=+$ here would be $P\left(X_{i}=+\mid S\right)=30 /(30+15)=2 / 3$.

In general, if a variable $X$ 's prior distribution is $X \sim \operatorname{Dir}\left(\alpha_{1}, \ldots, \alpha_{w}\right)$, then

$$
P(X=i)=\frac{\alpha_{i}}{\sum_{k} \alpha_{k}}
$$

If we then observe a sample $S$ that includes $a_{i}$ instances of $X=i$, then $X$ 's posterior distribution remains a Dirichlet, with new parameters

$$
X \mid S \sim \operatorname{Dir}\left(\alpha_{1}+a_{1}, \ldots, \alpha_{w}+a_{w}\right) .
$$

In the formal description above, a probe of the form $x_{i}^{(j)}$ specifies the feature to probe $\left(X_{i}\right)$ and the specific instance in the tableau (instance $\mathrm{j}$ ) on which to perform the probe. However, because of our Naïve Bayes assumption, we can treat all instances with the same class label identically. Thus, rather than querying specific instances, we only consider probes of the form $(i, y)$ that request the $i$ th feature of a randomly chosen instance in the tableau whose class label is $y$. (By convention, this process selects the value of an $(i, y)$ feature-value that has not been seen before.)

\footnotetext{
${ }^{2}$ The sparsity of the data means the obvious frequentist approach of using simple frequencies is problematic.

3 Note that Naïve Bayes models often produce good classifiers even for datasets that violate this assumption.
} 


\subsection{Complexity Results}

Madani et al. 4 proves the following much simpler task is NP-hard: Given a set of coins with known prior distributions and a fixed total number of flips, decide when to flip which coin to decide which coin has the highest head probability. Our framework inherits that negative result. (Identify each coin $f_{i}$ with a binary feature, whose head probability corresponds to the probability the class is true, given $f_{i}$ is true, $P\left(c=+\mid f_{i}=+\right)$; we also let $P\left(c=+\mid f_{i}=-\right)=0$ for all features.) In addition, 1] shows that computing the best active classifier is NPhard in general, even if we know the entire distribution. Our framework inherits that negative result as well.

\section{The Optimal Policy}

As our problem is a finite Markov Decision Process, there exists a deterministic optimal policy for spending the learning budget such that the expected total $\left(\right.$ expected $\left._{2}\right)$ misclassification errol $^{4}$ of the final bounded active classifier is minimized. Mathematically, the optimal learning policy is the one that minimizes:

$$
\sum_{i \in \text { Outcomes }} P(i) \sum_{\mathbf{x}, y} P(\mathbf{x}, y \mid i) L\left(B A C^{*}(\mathbf{x}), y\right)
$$

where each "outcome" corresponds to a state in which our learning budget has been fully exhausted and has resulted in posterior Dirichlet distributions over the feature values.

Such a policy can be computed via a bottom-up dynamic program. Unfortunately, the number of outcomes (and hence the computational complexity) has a prohibitive lower bound:

Proposition 1. [5] Let $\left|X_{i}\right|$ denote the domain size of feature $X_{i},|S|$ denote the number of classes, $t=|S| \sum_{i}\left|X_{i}\right|-1$, and each feature has unit cost. Then the bottom-up dynamic program must compute the value of $\Omega\left(\left(\frac{b_{L}+t}{b_{L}}\right)^{b_{L}}\left(\frac{b_{L}+t}{t}\right)^{t} \frac{1}{\sqrt{t}}\right)$ outcomes.

We have considered improving upon this naïve dynamic program by reducing the number of subproblems that must be solved. Below we show an interesting way to achieve this reduction by exploiting the equivalence of two "permuted" states under the conditional independence assumption.

Definition 1. A proper permutation for a feature $X_{i}$ with $w$ domain values is a bijective function $f:[1, w] \rightarrow[1, w]$ that applies the same reordering of the $w$ parameters for every Dirichlet distribution on $X_{i}$.

${ }^{4}$ The first expectation ${ }_{1}$ is over the set of possible Dirichlet distributions produced

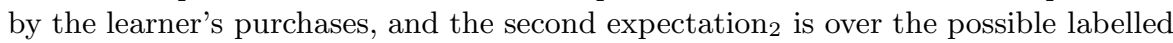
instances $(\mathbf{x}, y)$ that can occur given the resulting Dirichlets. 
Example 1. Let

$$
\left(X_{i} \mid Y=0\right) \sim \operatorname{Dir}(4,2,7), \quad\left(X_{i} \mid Y=1\right) \sim \operatorname{Dir}(3,8,5)
$$

Then a proper permutation for feature $X_{i}$ is:

$$
\left(X_{i} \mid Y=0\right) \sim \operatorname{Dir}(7,2,4), \quad\left(X_{i} \mid Y=1\right) \sim \operatorname{Dir}(5,8,3) .
$$

Proposition 2. [5] Assume the Naïve Bayes assumption holds, and identify a "state" of our problem by the value of $b_{L}$ and the set of Dirichlets over the feature-class pairs. Consider any two states $A$ and $B$, that have equal values of $b_{L}$ and are such that the Dirichlets of $A$ can be made equal to the Dirichlets of $B$ by specifying a set of $r$ proper permutations, one for each feature $X_{i}$. Under these conditions, the expected value of state $A$ is equal to the expected value of state $B$ when following an optimal policy, and the optimal action to take from state $A$ is the optimal action to take from state $B$.

This proposition allows us to improve the naïve dynamic program by reusing the computed value of a state $A$ for properly permuted versions of $A$. The realtime improvement using Proposition 2 is shown in Table 1 . In the last case, the naïve dynamic program ran out of memory after more than two hours, while our improved version finished properly in under an hour. Unfortunately such improvements are not sufficient to remove the exponential complexity of the dynamic program (recall that this task is NP-complete); therefore, we consider the following more tractable, suboptimal approaches.

Table 1. Reduction in computation time using Proposition 2

\begin{tabular}{cccccc}
\hline$b_{L}$ & $b_{C}$ & Features Domain Size & Naïve & Improved \\
\hline 2 & 4 & 6 & 4 & $161 \mathrm{sec}$ & $65 \mathrm{sec}$ \\
3 & 2 & 4 & 3 & $888 \mathrm{sec}$ & $432 \mathrm{sec}$ \\
4 & 3 & 4 & 3 & $8280 \mathrm{sec}$ & $3360 \mathrm{sec}$ \\
\hline
\end{tabular}

\section{Algorithms}

This section summarizes a number of "budgeted bounded-active-classifier learners". We focus on only the data collection part of the algorithms; after collecting $\$ b_{L}$ worth of feature-values, each of the algorithms then passes its learned (posterior) Dirichlet distributions to a dynamic program that produces the BAC* in (2).

\subsection{Round Robin (RR)}

This obvious algorithm simply purchases complete instances until its budget $b_{L}$ is exhausted. It draws examples randomly, and so expects to have collected data about members of each class $y$ in proportion to $P(Y=y)$. If there are $r$ unit-cost features, we expect to know everything about roughly $b_{L} / r$ instances. Notice RR implicitly assumes all features are equally valuable in learning the target concept. 


\subsection{Biased Robin (BR)}

A more selective approach than Round Robin is to purchase a single feature and test whether or not its observed value has increased some measure of quality. The Biased Robin algorithm is more selective than RR, continually purchasing feature $X_{i}$ as long as it improves quality, and otherwise moving to feature $X_{i+1}$ (and of course looping back to $X_{1}$ after $X_{r}$ ). There are several choices for how to measure quality or loss; see Sect. 5. Of course, BR must also specify a class $y$ from which to purchase its desired feature, and it does this by drawing from the class distribution $P(Y=y)$ on each purchase. As further motivation for this algorithm, [6] found it to be one of the best approaches for budgeted learning of a passive Naïve Bayes classifier, albeit with a different loss function. This method also corresponds to the "Play the Winner" approach discussed in [7.

\subsection{Single Feature Lookahead (SFL)}

One would always like to avoid wasting purchases on poor features, especially when faced with a limited learning budget. This motivates a prediction-based approach, which uses a loss function to estimate the expected loss incurred after making a sequence of purchases of a single, specified feature.

SFL uses this prediction based approach, and controls the level of myopia or "greediness" involved by providing an additional parameter, $d=$ the lookahead depth. With a lookahead depth of $d$, SFL calculates the expected loss of spending its next $\$ d$ sequentially purchasing feature $i$ of instances of class $j$. That is, if $S$ denotes our current set of Dirichlets and $S^{\prime}$ denotes the Dirichlets after spending $\min \left(\$ d, \$ b_{L}\right)$ purchasing feature $X_{i}$ of $Y=j$ instances, then the expected loss for $(i, j)$ is:

$$
S F L(i, j)=\sum_{S^{\prime}} P\left(S^{\prime} \mid S\right) \operatorname{Loss}\left(S^{\prime}\right) .
$$

SFL determines the feature-class pair $(i, j)$ with lowest expected loss, then purchases the value of this best $(i, j)$ feature for one instance, and updates the Dirichlets based on the observed outcome of that purchase (and reduces the available remaining budget). It then recurs, using (6) to compute the score for all feature-class pairs in this new situation - with its updated Dirichlets and a smaller budget. This process repeats until the learning budget is exhausted. The lookahead depth $d$ can be set based on the computational resources available. If only the next one purchase is considered, then this reduces to the (1-step) greedy algorithm. SFL was originally used in two previously investigated variants of the budgeted learning problem [6]8].

\subsection{Randomized SFL (RSFL)}

Our experiments show that the SFL algorithm often spends the majority of its probes purchasing a single discriminative feature-class pair and neglects to explore other potentially good features. This property can be problematic, particularly when a dataset contains several discriminative features that can jointly 
yield a more accurate BAC than any single feature by itself. The Randomized Single Feature Lookahead algorithm (RSFL) alleviates this problem by increasing exploration among the best looking feature-class pairs. The RSFL algorithm is very similar to SFL, as it too calculates the expected loss in (6) for each feature-class pair. However, rather than deterministically purchasing the pair with the best SFL score, RSFL considers the best $K$ feature-class pairs and for each feature-class pair $(i, j)$ in this set, it chooses to purchase feature $i$ of class $j$ with probability:

$$
\frac{\exp \frac{-S F L(i, j)}{\tau}}{\sum_{i, j} \exp \frac{-S F L(i, j)}{\tau}}
$$

Here, $\tau$ is a temperature controlling exploration versus exploitation. Although we set $\tau$ to one throughout this paper, we include it in (7) to show the relationship to the Gibbs distribution. After experimenting with various values for the number of feature-class pairs, $K$, we found that $K=$ (number of classes) $\times b_{c}$ seemed to perform well, particularly when the learning budget was not much greater than the number of features.

\section{Loss Functions}

As mentioned earlier, several of our algorithms rely on a loss function

$$
\text { Loss : }\{\text { Dirichlet distributions over features }\} \rightarrow \mathbb{R}
$$

that attempts to measure the quality of a given probability distribution. After experimenting with several different choices of loss functions, we found Conditional Entropy Loss and Depth 1 BAC Loss to be effective 5

Conditional Entropy measures the uncertainty of the class label $Y$ given the value of a feature $X_{i}$ :

$$
-\sum_{x} P\left(X_{i}=x\right) \sum_{y} P\left(Y=y \mid X_{i}=x\right) \log _{2} P\left(Y=y \mid X_{i}=x\right) .
$$

The Biased Robin algorithm uses (9) before and after the purchase of feature $X_{i}$ to determine whether the purchase improved the ability of $X_{i}$ to predict the class $Y$.

On the other hand, other algorithms (SFL, RSFL, and greedy) use

$$
\min _{i} \sum_{x} P\left(X_{i}=x\right) \min _{y}\left(1-P\left(Y=y \mid X_{i}=x\right)\right)
$$

which calculates the expected misclassification error of the best Depth 1 BAC. Since BR needs to detect small changes in a distribution, it tends to perform better with the more sensitive conditional entropy calculation in (9).

${ }^{5}$ The obvious loss function is just to use (2) to compute the expected error of the optimal BAC. However, since loss functions can be called several times on a single purchase, the computational expense of computing (2) is prohibitive. 


\section{Experimental Results}

To compare the algorithms, we tested their performance on several datasets from the UCI Machine Learning Repository [9]. We used supervised entropy discretization [10] to discretize datasets with continuous values. Each dataset was then randomly partitioned into five folds. The algorithms were run five times, and on each run a single fold was set aside for testing, while the remaining four were available for purchasing. For each algorithm, we used the average value of these five runs as the algorithm's misclassification error on the whole dataset. We repeated this process 50 times to reduce the variance and get a measure of the average misclassification error. Thus, each point in the graphs that follow represents 50 repetitions of five-fold cross validation.

In the first set of experiments, all features have unit cost and the datasets contain some irrelevant features. We set the classifier's budget to $b_{c}=3$, as this is large enough to allow several features to be used, but small enough to keep computations tractable. All Dirichlets parameters are uniformly initialized to 1. For reference, each graph also includes a gold standard "All Data" algorithm, which is allowed to see the entire dataset, and thus represents the best that one can do using the Naïve Bayes assumption on the data.

Figure 1 shows the performance of the algorithms on the Glass Identification dataset: a binary class problem with nine features whose domain sizes vary between one and three. The four features that have a domain size of one represent irrelevant information that any learning algorithm (especially one under a constraining budget) should avoid. Both RSFL and BR learn better than the obvious RR algorithm for all learning budgets considered. In fact, we found the optimal $b_{C}=3$ BAC produced by the "All Data" algorithm involves four different features, and these four features are precisely the ones that RSFL and BR purchase heavily during learning. This is in contrast to the RR purchasing behaviour that spends equally on all features, despite their unequal predictive power. Finally, SFL and Greedy spend their entire budget on only one or two features during learning, which accounts for their low accuracy BACs.

The Breast Cancer dataset contains ten features, only one of which is irrelevant to the concept. This dataset is particularly interesting because nearly all its features are good predictors, but three features have markedly lower conditional entropy than the rest. To produce the lowest error BAC, the learning algorithms must discover the superiority of these three features. We find RSFL does exactly this, spending $20 \%, 21 \%$, and $32 \%$ of its budget respectively on the three strong features. In comparison, RR spends $10 \%$ of its budget on every feature which makes it much more difficult for it to separate the top features from the rest. BR also performs better than RR for all learning budgets considered.

The next set of experiments, shown in Fig. 2, considers datasets without any irrelevant features. The Iris dataset has only four features and is a three class problem. Given that all four features are relevant, and that $b_{C}=3$ in this experiment, the optimal BAC requests every feature at some point in its tree. With only four features to consider, RSFL is able to test them all effectively and 

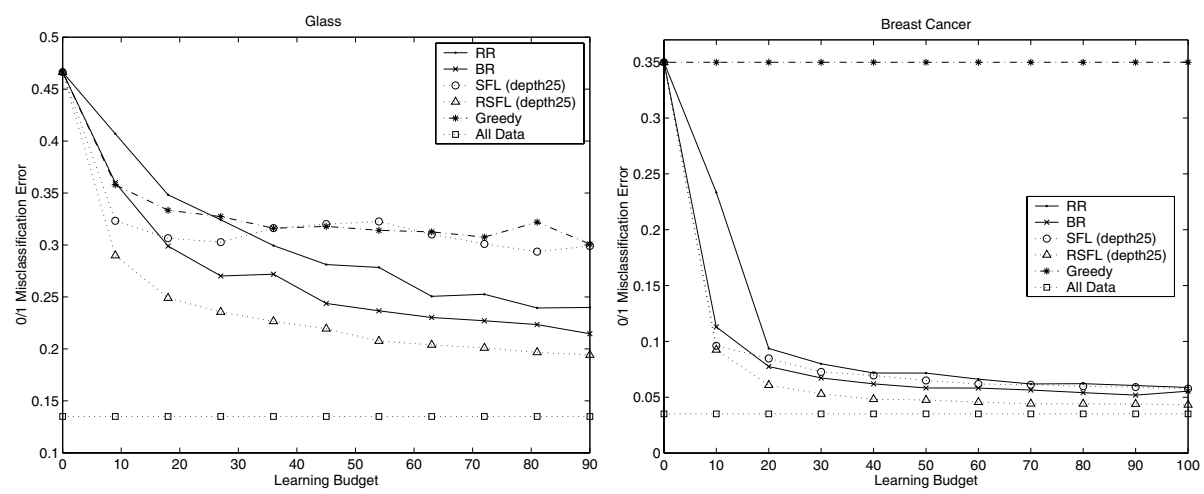

Fig. 1. Identical costs and some irrelevant features - RSFL and BR outperform RR
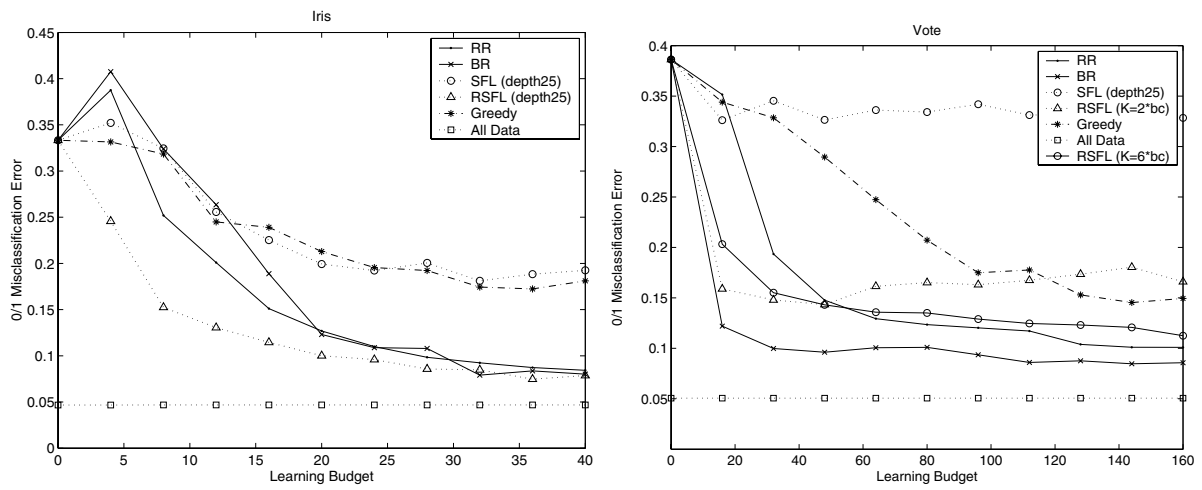

Fig. 2. Identical costs, no irrelevant features - RR still suboptimal

produce better BACs than RR for all budgets considered. BR is also competitive with RR, except at some of the very low budgets where BR's exploration model prevents it from ever investigating some of the features.

Figure 2 (right) shows another binary class problem, the Vote dataset, that contains 16 features. Many of these features have similar (high) predictive power. Once again we see that both RSFL and BR beat RR when the learning budget is small. RSFL asymptotes after about 50 purchases — it spends its budget finding a few strong features quickly and outputs a fairly low error BAC. As expected, at larger budgets RR collects enough information on every feature to find many more suitable candidates for its BAC than RSFL can. The graph shows that one can improve the performance of RSFL by increasing the number of top feature-class pairs that RSFL considers on this dataset. We also observe that BR's exploration model is particularly well suited to this task because it is able to collect information on every feature at larger budgets, which is crucial on a dataset such as Vote with a large number of predictive features. 

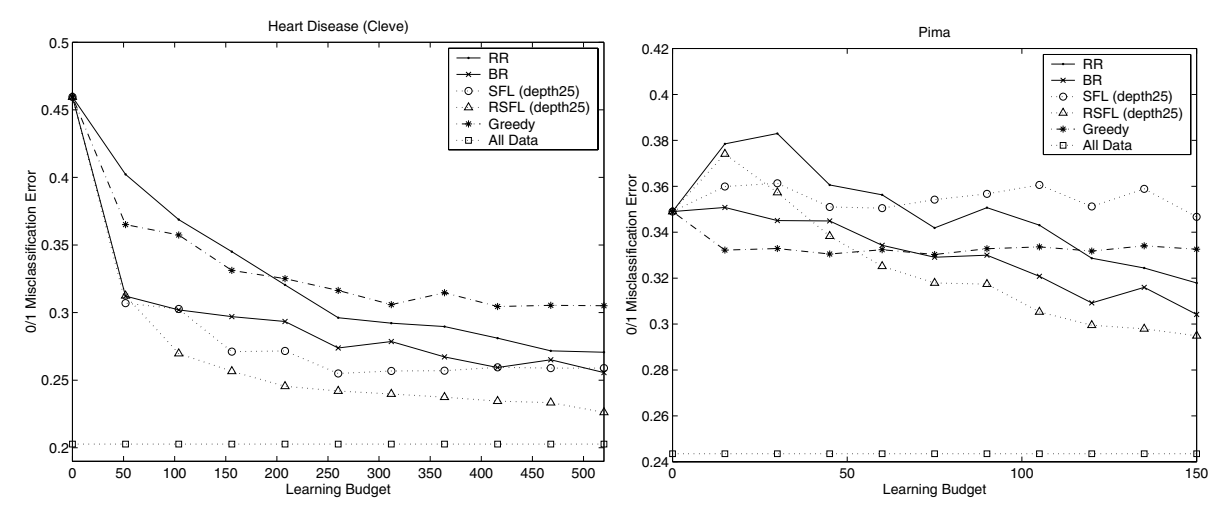

Fig. 3. Different feature costs - RSFL and BR dominate RR

Our final set of experiments involved datasets where the features differed in cost. Both the Heart Disease dataset and the Pima Indians dataset have known cost data [9], which we used in our tests. The scaled Heart Disease costs range from $\$ 1$ to $\$ 7$, and our tests are run with $b_{c}=\$ 7$. This dataset represents the worst case for RR, because the irrelevant features happen to be the most expensive ones. In fact, RSFL achieves the same error rate after $\$ 100$ that RR takes $\$ 500$ to reach. In the Pima dataset, feature costs are between $\$ 1$ and $\$ 5$, and we set $b_{c}=\$ 5$. The two irrelevant features have cost $\$ 1$, and the single best feature is $\$ 4$. Once again, BR and RSFL dominate RR for all budgets considered.

\section{Related Work}

There are a number of different senses of "costs" in the context of learning [1]. Our research considers two of these: the costs paid by the learner to acquire the relevant information at training time to produce an effective classifier and also the costs paid by the classifier, at performance time, to acquire relevant information about the current instance. We impose hard constraints on the total cost of tests that can be performed per instance, and on the expenses paid by the learner.

Many existing (sub)fields, such as active learning [12] and experimental design 13. (as well as earlier results such as [6]) focus on only the first of these costs - e.g., bounding how much the learner can spend to produce an accurate passive classifier. In addition, many of these systems request the class label for an otherwise completely specified instance. Thus they require only a single quantity per instance. Our problem is the complement of this: class labels are known but feature information must be purchased. Unlike most of the other models, this means our work may need to consider the correlations amongst the many unknown properties of an instance. Other results seeking to reduce the sample complexity for learning include decision theoretic subsampling [14, on-line stopping rules [15], progressive sampling [16], and active feature value acquisi- 
tion [17. We note that these techniques differ from our approach because we place a firm prior budget on the learner's ability to acquire information, while these approaches typically allow the learner to purchase until some external stopping criteria (for instance, accuracy) is satisfied.

Weiss and Provost [18] recently explored a problem related to one that we encounter in our overall framework: how to represent the class distribution when only a firm budget of $n$ training examples can be used. As discussed in Sect. 4 , our algorithms select which class to probe in different ways (e.g. performing lookahead (SFL, Greedy), drawing from the true class distribution (RR and $\mathrm{BR}$ ), or combining lookahead with a Gibbs distribution (RSFL)).

As for the costs paid by the classifier at performance time, both [19] and [1] attempt to produce a decision tree that minimizes expected total cost. However, neither work assumes an a priori resource bound on the learner, thereby allowing for unconstrained amounts of training data with which to build these classifiers. Again, our work makes the more realistic assumption that if data costs money at performance time, it very likely costs money at learning time as well.

Finally, we can view our model as a (fixed horizon, partially observable) Markov Decision Process (MDP) [20. We note that although the MDP formulation is theoretically clear, it has not yielded strong results in our experiments due to the dimensionality and lack of suitable features for function approximation; see [21. A simpler version of our problem also exists in the MDP framework [8], and the results of that work motivate several of the policies that we adapt for budgeted learning of bounded active classifiers.

\section{Conclusions}

Many standard learning algorithms implicitly assume the features are always available for free, to both the learner at "training time" and later the classifier, at "performance time". This paper extends those systems by explicitly considering these costs, at both training and performance time. It introduces the formal framework for budgeted learning a bounded active classifier, and presents some complexity results. We also propose a more efficient way to implement the optimal algorithm, which we prove works effectively. Moreover, this paper motivates and defines a variety of tractable learning strategies and shows they work effectively on various types of data - both with identical and with different feature costs. In particular, we demonstrated that our proposed strategies can often do much better than the obvious algorithm - "Round Robin" - especially when training data is limited.

\section{References}

1. Greiner, R., Grove, A.J., Roth, D.: Learning cost sensitive active classifiers. Artificial Intelligence (2002)

2. Dobkin, D., Gunopoulos, D., Kasif, S.: Computing optimal shallow decision trees. In: International Workshop on Mathematics in Artificial Intelligence. (1996) 
3. Heckerman, D.: A tutorial on learning in bayesian networks. In: Learning in Graphical Models. The MIT Press (1999)

4. Madani, O., Lizotte, D.J., Greiner, R.: Active model selection. Technical report, University of Alberta (2004)

5. Website: http://www.cs.ualberta.ca/ greiner/RESEARCH/blweb.html (2005)

6. Lizotte, D.J., Madani, O., Greiner, R.: Budgeted learning of naive-bayes classifiers. In: Proceedings of Uncertainty In Artificial Intelligence. (2003)

7. Robbins, H.: Some aspects of the sequential design of experiments. Bulletin of the American Mathematical Society (1952)

8. Madani, O., Lizotte, D.J., Greiner, R.: Active model selection. In: Proceedings of Uncertainty in Artificial Intelligence. (2004)

9. S. Hettich, C.B., Merz, C.: UCI repository of machine learning databases (1998)

10. Fayyad, U., Irani, K.: Multi-interval discretization of continuous-valued attributes for classification learning. In: IJCAI. (1993)

11. Turney, P.: Types of cost in inductive concept learning. In: Workshop on cost sensitive learning (ICML). (2000)

12. Cohn, D.A., Ghahramani, Z., Jordan, M.I.: Active learning with statistical models. In: Advances in Neural Information Processing Systems. (1995)

13. Chaloner, K., Verdinelli, I.: Bayesian experimental design: A review. Statistical Science (1995)

14. Musick, R., Catlett, J., Russell, S.: Decision theoretic subsampling for induction on large databases. In: International Conference on Machine Learning. (1993)

15. Schuurmans, D., Greiner, R.: Sequential pac learning. In: COLT. (1995)

16. Provost, F., Jensen, D., Oates, T.: Efficient progressive sampling. In: International Knowledge Discovery and Data Mining Conference. (1999)

17. Melville, P., Saar-Tsechansky, M., Provost, F., Mooney, R.: Active feature-value acquisition for classifier induction. In: ICDM. (2004)

18. Weiss, G.M., Provost, F.: Learning when training data are costly: the effect of class distribution on tree induction. Journal of Artificial Intelligence Research (2003)

19. Turney, P.: Cost-sensitive classification: empirical evaluation of a hybrid genetic decision tree induction algorithm. Journal of Artificial Intelligence Research (1995)

20. Russell, S., Norvig, P.: Artificial Intelligence: A Modern Approach. Prentice Hall (2002)

21. Kapoor, A., Greiner, R.: Reinforcement learning for active model selection. In: Utility Based Data Mining Workshop (KDD). (2005) 